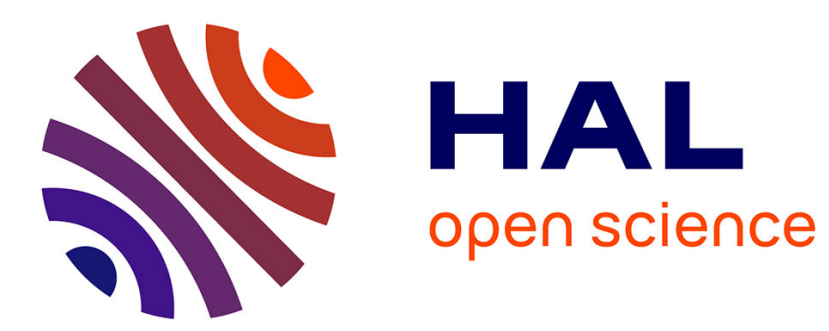

\title{
An end to end approach to the resequencing problem
}

François Baccelli, E. Gelenbe, B. Plateau

\section{To cite this version:}

François Baccelli, E. Gelenbe, B. Plateau. An end to end approach to the resequencing problem.

RR-0097, INRIA. 1981. inria-00076464

\section{HAL Id: inria-00076464 \\ https://hal.inria.fr/inria-00076464}

Submitted on 24 May 2006

HAL is a multi-disciplinary open access archive for the deposit and dissemination of scientific research documents, whether they are published or not. The documents may come from teaching and research institutions in France or abroad, or from public or private research centers.
L'archive ouverte pluridisciplinaire HAL, est destinée au dépôt et à la diffusion de documents scientifiques de niveau recherche, publiés ou non, émanant des établissements d'enseignement et de recherche français ou étrangers, des laboratoires publics ou privés. 


\section{Rapports de Recherche}

$$
\mathrm{N}^{\circ} 97
$$

\section{AN END TO END APPROACH TO THE RESEQUENCING PROBLEM}

7. 4.6. 37. - E. 3.5. (5) $y$ 3.t. t.

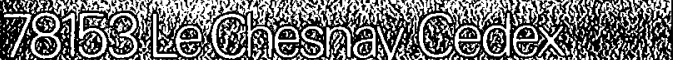
1.

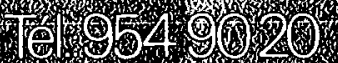

\author{
François BACCELLI \\ Erol GELENBE \\ Brigitte PLATEAU
}




$\begin{array}{lll}\text { François BACCEILI } & \text { Erol GELENBE } & \text { Brigitte PLATEAU } \\ \text { INRIA } & \text { LRI - Băt. 490 } & \text { IRI - Bât. 490 } \\ \text { Domaine de Voluceau } & \text { Univ. Paris-Sud } & \text { Univ. Paris-Sud } \\ \text { BP 105 - Rocquencourt } & 91405 \text { Orsay } & 91405 \text { Orsay } \\ \text { 78153 Le Chesnay } & & \end{array}$

\section{Abstract :}

The resequencing or reserialisation problem is of basic interest in distributed systems and computer communication systems. This is because a flow of packets, messages or updates entering a communication system in chronological order fram the same port or fram different ports may be disondered. The receiving port must then insure that these objects are resequenced in the appropriate order before they are fed to the output of the system. In this paper we analyze the end-to-end delay incurred by objects traversing such a system, including both the disordering delay, the delay introduced by the resequencing algorithm and the delay due to the output server at the receiving port. The analysis is carried out via spectral factorization methods.

\section{Résumé :}

Ies phénomènes de reséquencement sont de première importance dans les systèmes à contrôle réparti (bases de données réparties, réseaux de processeurs, réseaux de télécommunication). En effet, la répartition du contrôle ne permet pas, en règle générale, de préserver l'ordre chronologique des émissions de messages (demandes de mise à jour, messages de synchronisation, paquets de données) pouvant avioir lieu en chacun des noeuds du système à destination d'un noeud terminal donné. Ies algorithmes de reséquencement implémentés en ce dernier noeud sont destinés à restaurer l'ordre chronologique des messages arrivant au processeur terminal. Dans cette étude, nous analysons le délai de bout en bout subi par les messages traversant un tel système : délai de transmission, délai de reséquencement, délai de traitement. Des équations récursives sont établies qui permettent de réduire l'analyse de ce délai à des problèmes de factorisation spectrale étudiés en détail sur des exemples. 


\section{AN END TO END APPROACH TO THE}

RESEQUENCING PROBLEM

\section{INTRODUCTION}

The resequencing problem is a fundamental issue in networks and in distributed systems. Let us first give an abstract statement and then provide examples of some practical occurrences of the problem.

Consider a sequence of objects $\left\{\phi_{n}\right\}_{n \in N^{\prime}}$ where $\mathbf{N}$ denotes the set of all non-negative integers. They enter a communication system at instants $\left\{a_{n}\right\}_{n \in \mathbb{N}}$ where $a_{n}$ corresponds to $\phi_{n}$.

Each $\phi_{n}$ is then delayed by same time $D_{n}, n \in \mathbb{N}$. Thus at the output point of the system the objects appear at instants $\left\{a_{n}+D_{n}\right\}_{n \in \mathbb{N}}$ : these instants are not necessarily in chronological order any more (i.e. it is possible that $a_{n}+D_{n}>a_{\ell}+D_{\ell}$ for $\ell>n$ ). 
These objects are then processed by the Resequencing Algorithm (RA) where $\phi_{n}$ will receive some service of duration $s_{n}$ and will then depart at time $d_{n}$. However this service can only be given in the same order as that of the external arrival instants ; i.e. , $\phi_{n}$ must be served after $\phi_{n-1}$ and before $\phi_{n+1}$, which does not necessarily coincide with the order of arrival to the RA. We would like to know what all this would cost, and in particular we would like to evaluate the total effective delay $\left(a_{n}-a_{n}\right)$.

This problem has multiple practical incarnations : let us now outline a few areas of application.

\section{I.1. Packet switching networks}

Packet switching networks are well known practical camputer communication systems [1] used to interconnect computer systems or subsystems by transmitting streams of data in the form of standardized packets. Thus packets belonging to the same message, or to the same logical stream (e.g. in the case of a file transfer) have to be reassembled at the receiving station in the proper onder, identical to the order at the transmitting station. Yet the packet switching network may not necessarily guarantee this particular order if it allows adaptive routing, or alternate routing, in order to handle congestion problems or temporary unavailability of certain routes. Therefore, the output nodes of the network may themselves insure proper packet reassembly before they provide an output ; in this case, the resequencing delay must be included in the end-to-end delay evaluation for the network.

\section{I.2. Distributed data bases}

Several consistency preserving mechanisms or algorithms $[2,3,4]$ for distributed data bases use a logical or physical time-stamping mechanism in onder to determine the order in which updates must be 
carried out on the data. For obvious reasons, updates do not necessarily arrive at the system sites in that order : these updates may originate at distinct nodes, thus they often arrive out of sequence even though the network may be able (and this too is not always the case) to transmit updates in sequence between a given pair of nodes. Thus the desequencing introduced by the system, and the corresponding resequencing problem, are basic issues in time-stamp oriented algorithms. It is this particular application which has motivated the first analyses of the problem $[4,5,7]$.

\section{I.3. The Mathematical problem}

Consider the system shown on Figure 1. The objects $\left\{\phi_{n}\right\}$ which enter into the system at instants $\left\{a_{n}\right\}$ and which depart (in order) at $d_{1} \leq d_{2} \leq \ldots \leq d_{n} \leq \ldots$ must be processed by the resequencing algorithm before being transferred to the output server queue. Notice that $S_{n}$ is a service time inside the RA.

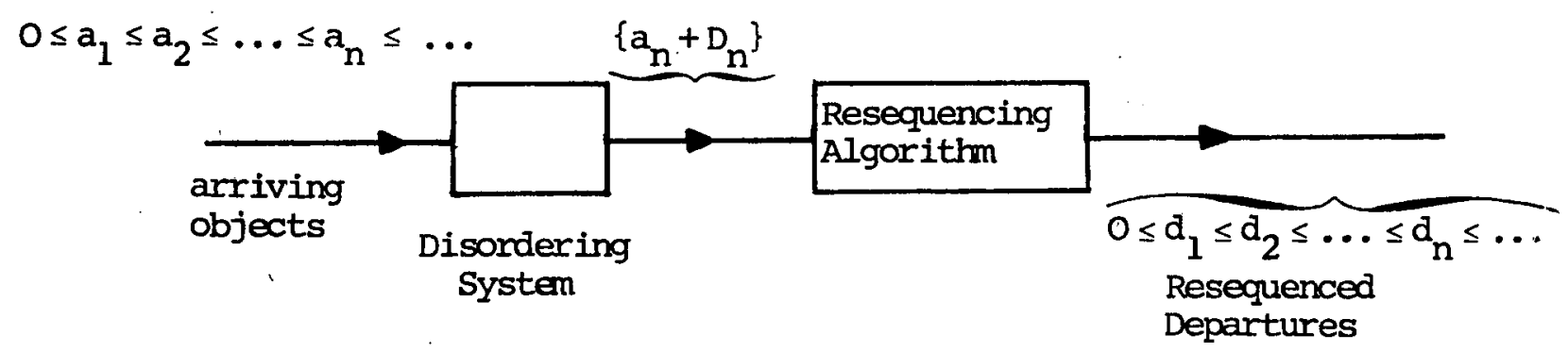

Figure 1 : Structure of the resequencing problem

Kamoun, Kleinrock and Muntz [5] have analyzed the distribution of the number of objects in the "Resequencing Algorithm" box of Figure 1 under the assumption that the arrival instants constitute a Poisson process, that the delays $\left\{D_{n}\right\}_{n \geq 1}$ are independent identically distributed (iid) randam variables of exponential distribution (this corresponds to the case when the disorder is provoked by an $M / M / \infty$ 
type system) and when the processing time is zero $\left(S_{n}=0\right)$. This analysis allows them also to obtain the average delay due to the resequencing algorithm. Harrus and Plateau [6] generalize this approach to the case where the delays $\left\{D_{n}\right\}$ are iid random variables of general distribution. Baccelli and znati [7] have considered an "approximate" model of the output queue in which the resequencing is represented by interruptions in the service mechanism. Finally Feuvre [8] has also developed an approximate approach by modelling it as a queue-length dependent bulk service mechanism.

In this paper we take a formal "end-to-end" approach to the problem, which allows to include the effect of the output service mechanism. We obtain certain "existence" type results with very weak assumption on the various variables involved, and then examine cases where the $\left\{D_{n}\right\}$ are, or are not, iid. our approach provides a complete solution to cases previously considered in $[5,6]$, and also allows analysis of a case where the delays $\left\{D_{n}\right\}$ have a form of dependency : this is an important extension for the following reason.

In the $\left\{D_{n}\right\}$ represent delays of successive packets or updates travelling through a network or a distributed system, delays experienced by successive packets will be independent only if the paths traversed by successive packets or updates are disjoint, or if the message traffic rate is very low. In other cases, same form of dependence will naturally exist.

\section{A WAITING TIME OR TOTAL DELAY EQUATION FOR THE RESEQUENCING} ALGORITHM

The resequencing algorithm (RA) operates as follows. It delays an object $\phi_{n}$ until all of the objects $\phi_{j}, j<n$, have been released by the RA. The RA will release objects sequentially, taking $s_{n}$ units of time to release $\phi_{n^{\prime}}$ only after $\phi_{n-1}$ has been released. 
Thus the RA may be viewed as a "real-time sorting algorith". We are interested in the end-to-end properties of this system, from the arrival instant of an object to its instant of release by the RA.

Remark 1. In the other models considered in the literature, and in particular the one treated by Kamoun, Kleinrock, Muntz [5] and Harrus, Plateau [6] the processing time $s_{n}$ of the resequencing algorith is not represented. Our basic model reduces to their's simply by setting $s_{n}=0, n \geq 1$.

Let us call $T_{n}=d_{n}-a_{n}$ the total delay separating the instant $a_{n}$ at which the object $\phi_{n}$ arrives to the system fram the instant $d_{n}$ at which it is released by the RA.

We may write

$$
T_{n}=D_{n}+S_{n}+W_{n}
$$

where $w_{n}$ is the time during which $\phi_{n}$ has to wait in the RA before it is serviced and released.

Lemma 1. We have $T_{1}=D_{1}+S_{1}$, and for $n \geq 2$ :

$$
T_{n}=\left\{\begin{array}{l}
D_{n}+S_{n} \text { if } D_{n}>T_{n-1}-\left(a_{n}-a_{n-1}\right) \\
D_{n}+S_{n}+\left[T_{n-1}-\left(a_{n}-a_{n-1}\right)-D_{n}\right], \text { otherwise }
\end{array}\right.
$$

Proof : $\phi_{n}$ will only be released after $\phi_{n-1}$ 's release instant which is

$$
a_{n-1}+T_{n-1}
$$

but obviously not before

$$
a_{n}+D_{n}+s_{n}
$$


Thus $\phi_{\mathrm{n}}$ cannot be processed by the RA before instant

$$
a_{n}+D_{n}
$$

Hence if

$$
a_{n}+D_{n}>a_{n-1}+T_{n-1}
$$

then $\phi_{\mathrm{n}}$ will be released at instant

$$
a_{n}+D_{n}+s_{n}
$$

yielding $T_{n}=D_{n}+S_{n}$. Otherwise, if

$$
a_{n}+D_{n}<a_{n-1}+T_{n-1}
$$

it follows that $\phi_{n-1}$ will wait in the RA for a duration of

$$
a_{n-1}+T_{n-1}-\left(a_{n}+D_{n}\right)
$$

and will only be released at

$$
D_{n}+S_{n}+\left[a_{n-1}+T_{n-1}-\left(a_{n}+D_{n}\right)\right]
$$

hence the result.

We shall write the formula of Ienma 1 in the more convenient form

(1) $\left\{\begin{array}{l}w_{1}=0 \\ w_{n}=\left[w_{n-1}-A_{n}+s_{n-1}-\left(D_{n}-D_{n-1}\right)\right]^{+}, n \geq 2\end{array}\right.$

where $W_{n}+S_{n}$ is the total time spent by $\phi_{n}$ in the $R A,[X]^{+}$denotes $\max [0, x]$ as usual, and $A_{n}=a_{n}-a_{n-1}$. 
Lemma 2. Consider the sequence $\left\{w_{n}\right\}_{n \geq 1}$ defined by equation (1). Define the randam variable $\xi_{n}$ by

$$
\xi_{n}=S_{n-1}-A_{n}-\left(D_{n}-D_{n-1}\right), n \geq 2
$$

so that we can write

(2) $\left\{\begin{array}{l}w_{1}=0 \\ w_{n}=\left[w_{n-1}+\xi_{n}\right]^{+}, \quad n \geq 2\end{array}\right.$

If $\left\{\xi_{n}\right\}_{n} \geq 2$ is a sequence of identically distributed (but not necessarily independent) random variables, then when $E\left[\xi_{n}\right]<0$ it follows that

$W_{n} \underset{P}{\rightarrow} W$ a proper randam variable

Proof : Notice that (2) is identical in form to the well known waiting time formula for the $G / G / 1$ queve. The result then follows directly (see for instance Borovkov [9]), since it is identical to the corresponding result for the $G / G / 1$ system.

Remark 2. If we view the $\mathrm{RA}$ as a "real-time sorting algorithm", then we see that the condition $E\left[\xi_{n}\right]<0$, or equivalently

$$
E\left[A_{n}\right]>E\left[S_{n-1}\right]+E\left[D_{n-1}-D_{n}\right] \text {, or } E\left[a_{n}+D_{n}\right]>E\left[a_{n-1}+D_{n-1}\right]+E\left[S_{n-1}\right]
$$

is a sufficient (and in fact necessary) condition for the sorting algorithm to keep up with its workload.

\section{THE CASE WHEN THE DELAY IS AN INDEPENDENT INCREMENT PROCESS}

\subsection{The waiting time}

In this section we make two assumptions which are quite common : the $\left(A_{n}\right)_{n \geq 1}$ and $\left(S_{n}\right)_{n \geq 1}$ are iid and mutually independent. 
Furthermore we assume that the $\left(D_{n}\right)_{n \geq 1}$ are not independent. We shall assume however that the $\left(D_{n}\right)_{n} \geq 1$ have independent increments. That 1s, that

$$
\begin{aligned}
& \theta_{n}=D_{n-1}-D_{n^{\prime}} n \geq 1 \\
& D_{0} \equiv 0
\end{aligned}
$$

where the $\left\{\theta_{n}\right\}_{n \geq 1}$ are 1 id and independent of the $\left(A_{n}\right)_{n \geq 1}\left(S_{n}\right)_{n \geq 1}$

Physically this is an appealing assumption since it states that $D_{n}$ is "nearly" equal to $D_{n-1}$, but differs from it by an "error term" given by $-\theta_{n}$.

Lemma 3. Under these assumptions, $\left\{w_{n}, n \geq 0\right\}$ is a Markov chain with state space $\mathbf{R}^{+}$satisfying the following transition equation

(1) $\left\{\begin{array}{l}w_{n}=\left[w_{n-1}+\xi_{n}\right]^{+} \\ n \geq 1\end{array}\right.$

Proof : this is simply because,$\xi_{n}$ is independent of the $\left\{w_{1}, 1 \leq n-1\right\}$.

Lemma 4. Under these assumptions, the Iaplace Stieltjes transform of the stationary distribution of the $W_{n}{ }^{\prime} s, F^{*}(s)$ satisfies the following functional equation if $E\left[\xi_{n}\right]<0$ :

(2) $\left\{\begin{array}{l}\text { For } \operatorname{Re}(s)=0 \\ {\left[1-A^{*}(s) \theta^{*}(s) \cdot S^{*}(s)\right] F^{*}(s)=U^{*}(s)}\end{array}\right.$

where $U^{\star}(s)$ is some unknown function which is analytic and bounded in the damain $\operatorname{Re}(s) \leq 0$.

Proof : Let $F(x)$ be $P\left[W_{n} \leq x\right]$ in steady state and $C(x)$ be $\mathrm{P}\left[\xi_{\mathrm{n}} \leq \mathrm{x}\right]$. We derive fram (1): 


$$
F(x)=\left\{\begin{array}{lc}
0 & x<0 \\
\int_{-\infty}^{x} F(x-y) d C(y) & x \geq 0
\end{array}\right.
$$

Define now

$$
G(x)=\left\{\begin{array}{lc}
0 & x \geq 0 \\
\int_{-\infty}^{x} F(x-y) d C(y) \quad x<0
\end{array}\right.
$$

We have, $\forall x \in \mathbb{R}$ :

$$
F(x)+G(x)=\int_{-\infty}^{x} F(x-y) d C(y)
$$

Taking the Fourier Stieltjes transforms of both sides of this equation yields :

$$
\left\{\begin{array}{l}
F^{\star}(s)=F^{\star}(s) A^{*}(-s) S^{\star}(s) \theta^{*}(s)+U^{*}(s) \\
\text { for } \operatorname{Re}(s)=0
\end{array}\right.
$$

where

$$
\left\{\begin{array}{l}
F^{*}(s) \triangleq \int_{0^{-}}^{\infty} e^{-s x} d F(x) \\
\text { for any } s \text { with } \operatorname{Re}(s) \geq 0
\end{array}\right.
$$

and

$$
\left\{\begin{array}{l}
U^{\star}(s) \triangleq-\int_{-\infty}^{O^{+}} e^{-s x} \int_{-\infty}^{x} F(x-y) d C(y) \\
\text { for any } s \text { with } \operatorname{Re}(s) \leq 0
\end{array}\right.
$$

Notice that $F^{*}(s)$ (resp $U^{*}(s)$ ) is analytic and bounded in the domain $\operatorname{Re}(s) \geq 0$ (resp $\operatorname{Re}(s) \leq 0)$.

Hence the analytical solution will be obtained by the spectrum factorization of the function 


$$
\left[1-A^{*}(-s) \theta^{\star}(s) S^{*}(s)\right]
$$

In the special case where $\theta^{*}(s)$ may be factorized as

$$
\left\{\begin{array}{l}
\theta^{*}(s)=D^{+}(s) \cdot D^{-}(-s), \quad \operatorname{Re}(s)=0 \\
\text { where both } D^{+}(s) \text { and } D^{-}(s) \text { are analvtic in } \operatorname{Re}(s) \geq 0
\end{array}\right.
$$

then $A^{*}(-s) \theta^{*}(s) S^{*}(s)$ may be factorized as $\left(A^{*}(-s) D^{-}(-s)\right)\left(S^{*}(s) D^{+}(s)\right)$ so that the problem reduces to the analysis of the GI/GI/1 queue with $A^{*}(s) \cdot D^{-}(s)$ (resp $s^{*}(s) \cdot D^{+}(s)$ ) interarrival (resp service time) distribution.

As an example, we now analyze the case where the delay increments have a bilateral exponential distribution function and the arrival process is poisson of parameter $\lambda$. (However, the techniques developed in the proofs may be generalized to any bilateral coxian distribution for the increments and coxian distribution for the interarrival times).

Theorem 5. In the case where $\theta^{*}(s)=\frac{\theta^{2}}{\theta^{2}-s^{2}}, \operatorname{Re}(s)=0$,

$$
\begin{aligned}
& \text { If } \lambda E[S]<1, \text { then } \\
& \exists F(x)=\lim _{n \rightarrow \infty} P\left[W_{n} \leq x\right] \text {, with Iaplace Stieltjes transform }
\end{aligned}
$$

(3) $F^{*}(s)=\frac{\theta}{\zeta} \cdot(1-\lambda E[S]) \cdot \frac{s(s+\theta)(s-\zeta)}{(\lambda-s)\left(\theta^{2}-s^{2}\right)-\lambda \theta^{2} s^{*}(s)}$

where $\zeta$ is the unique strictly positive real solution of the equation

$$
(\lambda-s)\left(\theta^{2}-s^{2}\right)=\lambda \theta^{2} s^{*}(s)
$$

Proof : $:$ Define

$$
\begin{aligned}
& R(s)=(\lambda-s)(\theta-s) F^{*}(s)-\lambda F^{*}(s) S^{*}(s) \cdot \frac{\theta^{2}}{\theta+s} \\
& \text { for } \operatorname{Re}(s) \geq 0 \\
& L(s)=U^{*}(s) \quad(\theta-s) \cdot(\lambda-s) \\
& \text { for } \operatorname{Re}(s) \leq 0
\end{aligned}
$$


We derive from (5) that $R(s)$ and $L(s)$ are equal for $\operatorname{Re}(s)=0$. Thus, $R(s)$ is the analytic contimuation of $L(s)$. We denote as $G(s)$ this unique function which is analytic in the whole complex plane.

$$
G(s)= \begin{cases}R(s) & \text { for } \operatorname{Re}(s) \geq 0 \\ L(s) & \text { for } \operatorname{Re}(s) \leq 0\end{cases}
$$

Let $\mathrm{H}(\mathrm{s})$ be

$$
H(s) \triangleq \frac{G(s)-G(\theta)}{s-\theta}
$$

and $I(s)$ be

$$
I(s) \triangleq \frac{H(s)-H(\lambda)}{s-\lambda}
$$

I(s) is analytic in the whole complex plane. Furthermore, I(s) is bounded for $\operatorname{Re}(s) \leq 0$ since in this damain :

$$
I(s)=-\frac{H(\lambda)}{s-\lambda}-\frac{G(\theta)}{(s-\lambda)(s-\theta)}+U^{*}(s)
$$

$I(s)$ is also bounded for $\operatorname{Re}(s) \geq 0$ since in this domain :

$$
\left\{\begin{array}{l}
I(s)=F^{*}(s)+\frac{\frac{E[s]-E[\theta]}{s-\theta}-\frac{E[\lambda]-E[\Theta]}{\lambda-\theta}}{s-\lambda} \\
\text { where } E(s) \triangleq-\lambda F^{*}(s) s^{*}(s) \cdot \frac{\Theta^{2}}{\theta+s}
\end{array}\right.
$$

Therefore, by Liouville's theorem I (s) is constant. Hence, $U^{*}(s)$, initially defined for $\operatorname{Re}(s) \leq 0$ may be continued for $\operatorname{Re}(s) \geq 0$ by the function

$$
U^{*}(s)=C+\frac{H(\lambda)}{s-\lambda}+\frac{G(\theta)}{(s-\lambda)(s-\theta)}
$$

Hence, we derive fram equation (2)

$$
F^{*}(s)=\frac{C\left(\theta^{2}-s^{2}\right)(\lambda-s)-H(\lambda)\left(\theta^{2}-s^{2}\right)+G(\theta)(\theta+s)}{\left(\theta^{2}-s^{2}\right)(\lambda-s)-\lambda \theta^{2} s^{*}(s)}
$$


The necessary condition

$$
\underset{s \rightarrow \infty}{F^{*}(s) \rightarrow F(0)}
$$

yields the relationship

$$
F(0)=C
$$

The necessary condition

$$
F^{*}(0)=1
$$

yields the following :

$$
\left\{\begin{array}{l}
\theta[F(0) \lambda-H(\lambda)]=-G(\theta) \\
\text { and (using I'Hospital's rule) } \\
F(0)=\frac{\theta}{\theta+\lambda}(1-\lambda E[S])-H(\lambda)
\end{array}\right.
$$

Thus, $F^{*}(s)$ may be writen as follows :

$$
F^{*}(s)=\frac{s(F(0) \cdot s-a)}{(\lambda-s)(\theta-s)-\lambda \theta s^{*}(s) \cdot \frac{\theta}{\Theta+s}}
$$

where

$$
a \triangleq \theta[1-\lambda E(S)]
$$

Let $N(s)$ (resp. $M(s)$ ) be the numerator (resp the dencminator) of the expression up there. $\mathrm{N}(\mathrm{s})$ has two real roots in $\operatorname{Re}(\mathrm{s}) \geq 0: 0$ and $\frac{a}{F(0)}$ (a is positive since $\lambda E[S]<1$ ). $M(s)$ has at least two real roots in $\operatorname{Re}(s) \geq 0$ : the first one is 0 and the existence of at least another real positive root is a consequence of :

$$
\left\{\begin{array}{l}
M(0)=0 \\
M(+\infty)=+\infty \\
M^{\prime}(0)=\theta(E[S] \lambda-1)<0
\end{array}\right.
$$

The analyticity of $F^{*}(0)$ for $\operatorname{Re}(s) \geq 0$ implies that $M(s)$ has at most one supplementary root in this domain, say $\zeta$ and that this root coinc1des with $\frac{a}{F(0)}$ completing the proof. 
Remark 3. A special case of interest is when $\theta \rightarrow \infty$ (ie $\left.D_{1} \Rightarrow D_{n}, n \geq 1\right)$. We derive then from theorem 5 :

$$
F^{*}(s)=\frac{s(\theta+s)[F(\theta) s-\theta(1-\lambda E[S])]}{(\lambda-s)\left(\theta^{2}-s^{2}\right)-\lambda \theta^{2} s^{*}(s)} \underset{\theta \rightarrow \infty}{\rightarrow} \frac{(1-\lambda E[S]) s}{s+\lambda\left(s^{*}(s)-1\right)}
$$

which coincides, as expected, with the waiting time transform in the M/G/1 system.

Remark 4. If $S_{n}=0, n \geq 1$ then

(6) $\zeta=\frac{\lambda+\sqrt{\lambda^{2}+4 \theta^{2}}}{2}$

Remark 5. Another quantity of interest is the average value $E[W]$ of the waiting time at steady state. After some algebra, we obtain as a consequence of theorem 5 :

(7) $E[W]=\frac{\Theta \cdot(1-\lambda E[S])(\theta-\zeta)+\lambda \cdot \zeta \cdot\left(1+\theta^{2} \frac{E\left[s^{2}\right]}{2}\right)}{(1-\lambda E[S]) \cdot \Theta^{2} \cdot \zeta}$

when $S_{n}=0$, this becomes :

(8) $E[w]=\frac{\theta^{2}+\zeta(\lambda-\theta)}{\theta^{2} \zeta}$

\subsection{The number in the system}

So far, we have been exclusively interested in properties of the delay incurred by an object in traversing the system. We shall now consider properties of the number of objects in the system.

Let $\mathrm{N}_{\mathrm{n}}$ denote the number of objects in the system just after the departure of $\phi_{n}$. Since the order of departure with respect to the order of arrival is first-in-first-out, this is simply the number of arrivals in the interval $\left.] a_{n^{\prime}} a_{n}+T_{n}\right]$. Since $T_{n}=D_{n}+W_{n}+S_{n}$, and because the arrival instants after $a_{n}$ do not depend on these quantities $\left(w_{n}\right.$ depends on the arrivals before $a_{n}$, see (1)), we can write 


$$
P\left[N_{n}=k\right]=\int_{0}^{\infty} \frac{(\lambda x)^{k}}{k !} e^{-\lambda x} d P\left[T_{n} \leq x\right]
$$

using the Poisson arrival assumption. We therefore have

(9) $\quad E\left[N_{n}\right]=\lambda E\left[T_{n}\right]=\lambda E\left[D_{n}\right]+\lambda E\left[W_{n}\right]+\lambda E\left[S_{n}\right]$

Theorem 6. Under the assumptions of theorem 5, the average number of objects $E[Q]$ waiting in the $R A$ in steady state is given by (10) $E[Q]=\lambda E[S]+\lambda \frac{\theta(1-\lambda E[S])(\theta-\xi)+\lambda \zeta\left(1+\theta \frac{E\left[S^{2}\right]}{2}\right)}{\zeta \cdot \theta^{2} \cdot(1-\lambda E[S])}$

Notice that, since $D_{n}$ and $w_{n}$ are not independent, it is not easy to obtain the distribution of $Q$. 


\section{ANALYTICAL SOLUTION FOR THE CASE OF INDEPENDENT DELAYS OF EXPONENTIAL DISTRIBUTION}

In [5], and later in [6], the case where the $\left\{D_{n}\right\}_{n \geq 1}$ are iid has been analyzed. The case when delays are exponential was treated in [5], while general independent delays were considered in [6]. In both cases, the number of customers in the R.A as well as analytical properties of the output process of the RA were computed (this last being considered as a bulk departure process). However, this approach leads to same difficulties for analyzing the end queue, since there is a correlation between the interarrival times and the bulk sizes. In the present section, we show that the end-to-end approach yields a tractable approach for analyzing the whole system including the end queue.

We first write the expression derived in lenma 1 in a form better suited to the present assumptions. Notice that when the $\left\{D_{n}\right\}_{n} \geq 1$ are iid, $\left\{w_{n}\right\}_{n} \geq 1$ given by $(1)$ is not a Markov chain anymore. Therefore, define :

$$
\mathrm{Y}_{\mathrm{n}} \equiv \mathrm{T}_{\mathrm{n}}-\mathrm{S}_{\mathrm{n}}, \mathrm{n} \geq 1
$$

where $T_{n}=D_{n}+S_{n}+W_{n}$; then we can write

$$
Y_{1}=D_{1}
$$

and for $n \geq 2$ :

$$
Y_{n}=\left\{\begin{array}{l}
D_{n} \text { if } \quad D_{n}>Y_{n-1}+S_{n-1}-A_{n} \\
Y_{n-1}+S_{n-1}-A_{n}, \text { otherwise }
\end{array}\right.
$$

or

$$
Y_{n}=\max \left[D_{n^{\prime}}\left(Y_{n-1}+S_{n-1}-A_{n}\right)\right], \quad n \geq 2
$$

as a direct consequence of Lenma 1. 
We shall assume that for all $n \geq 2$

$$
E\left[\xi_{n}\right]=E\left[s_{n-1}-A_{n}\right]<0
$$

(notice now that $E\left[D_{n}\right]=E\left[D_{n-1}\right]$ ) so that

$$
\mathrm{W}_{\mathrm{n}} \underset{\mathrm{P}}{\Rightarrow} \mathrm{W} \text { ( a proper randan variable) }
$$

since $Y_{n}=D_{n}+W_{n}$, we have also

(12) $Y_{n} \underset{P}{\Rightarrow} Y$ ( a proper randan variable)

\section{Denote}

$$
\begin{aligned}
& D(x)=P\left[D_{n} \leq x\right], \quad Y(x)=P[Y \leq x] \\
& C(x)=P\left[\xi_{n} \leq x\right]
\end{aligned}
$$

Fram (11) and (12) we can write

$$
\text { (13) } Y(x)= \begin{cases}D(x) \int_{-\infty}^{X} Y(x-z) d C(z), & \text { for } x>0 \\ 0 & \text { for } x \leq 0\end{cases}
$$

Now define

$$
U(x)=\left\{\begin{array}{l}
Y(x) / D(x) \text { if } x>0 \\
0 \text { otherwise }
\end{array}\right.
$$

since $w_{n} \geq 0$, it follows that for all $x \geq 0$

$$
\mathrm{P}[\mathrm{Y} \leq \mathrm{X}] \leq \mathrm{P}[\mathrm{D} \leq \mathrm{X}]
$$

Thus $Y(x) \leq 1$ for all $x \geq 0$; furthermore, since $Y$ and $D$ are proper randam variables

$$
\lim _{x \rightarrow \infty} U(x)=1
$$


Furthermore by (13), $U(x)$ satisfies

$$
U(x)= \begin{cases}\int_{-\infty}^{x} U(x-z) D(x-z) d C(z) & \text { if } x>0 \\ 0 & \text { otherwise }\end{cases}
$$

so that it is non-decreasing ; hence $U(x)$ is a probability distribution function ( $I n$ fact $U(x)$ is $P\left[Y_{n} \leq x \mid D_{n} \leq x\right]$ ). Define now

$$
U_{+}(x)=\left\{\begin{array}{l}
U(x) \text { if } x \geq 0 \\
0 \text { if } x<0
\end{array} \quad U_{-}(x)=\left\{\begin{array}{c}
0 \text { if } x \geq 0 \\
\int_{-\infty}^{x} U(x-z) D(x-z) d C(z) \text { if } x<0
\end{array}\right.\right.
$$

and their respective Laplace-Stieltjes transforms

$$
\begin{aligned}
& U_{+}^{*}(s)=\int_{-\infty}^{\infty} e^{-s x} d U_{+}(x), \operatorname{Re}(s) \geq 0 \\
& U_{-}^{*}(s)=\int_{-\infty}^{+\infty} e^{-s x} d U_{-}(x), \quad \operatorname{Re}(s) \leq 0
\end{aligned}
$$

Let $s^{*}(s), A^{*}(s)$, be the Laplace-Stieltjes transforms of the densities of $S$ and of $A$, respectively. We can write using (14) for all $x$ :

$$
U_{+}(x)+U_{-}(x)=\int_{-\infty}^{x} U(x-z) D(x-z) d C(z)
$$

so that when $\operatorname{Re}(s)=0$ :

$$
U_{+}^{*}(s)+U_{-}^{*}(s)=s^{*}(s) A^{*}(-s) K^{*}(s)
$$

where

$$
K^{*}(s)=\int_{0^{-}}^{\infty} d\left[U_{+}(x) D(x)\right] e^{-s x}, \operatorname{Re}(s) \geq 0 .
$$




\subsection{The case of Poisson arrivals and exponential distribution}

Equation (15) was obtained assuming that the $\left\{A_{n}\right\}_{n \geq 1}$ ' $\left\{D_{n}\right\}_{n \geq 1},\left\{S_{n}\right\}_{n \geq 1}$ are mutually independent sequences. To proceed further with the analytical solution we must take specific instances of the distribution. Assuming Poisson arrivals of parameter $\lambda$ and exponentially distributed delays of parameter $\mu$, we have :

$$
\left\{\begin{array}{l}
\mathrm{A}^{*}(\mathrm{~s})=\frac{\lambda}{\lambda+s} \\
\mathrm{D}^{*}(\mathrm{~s})=\frac{\mu}{\mu+1}
\end{array}\right.
$$

so that

$$
\begin{aligned}
\mathrm{K}^{*}(s) & =U_{+}^{*}(s)-U_{+}^{*}(s+\mu)+U_{+}^{*}(s+\mu) \cdot \frac{\mu}{s+\mu} \\
& =U_{+}^{*}(s)-\frac{s}{s+\mu} U_{+}^{*}(s+\mu), \quad \operatorname{Re}(s)=0
\end{aligned}
$$

yielding, using (15), for $\operatorname{Re}(s)=0$ :

$$
U_{+}^{\star}(s)+U_{-}^{*}(s)=s^{*}(s) \frac{\lambda}{\lambda-s}\left[U_{+}^{*}(s)-\frac{s}{s+\mu} U_{+}^{*}(s+\mu)\right]
$$

which is

$$
U_{-}^{*}(s)(\lambda-s)=U_{+}^{*}(s)(s-\lambda)+\lambda s^{*}(s)\left[U_{+}^{*}(s)-\frac{s}{s+\mu} U_{+}^{*}(s+\mu)\right]
$$

for $\operatorname{Re}(s)=0$.

Notice now that the Left-hand-side (IHS) of (16) is analytic for $\operatorname{Re}(s) \leq 0$, while its RHS is analytic for $\operatorname{Re}(s) \geq 0$, and by (16) they are equal for $\operatorname{Re}(s)=0$. Thus the RHS of (16) is the analytic continuation of the LHS

$$
u_{-}^{*}(s)(\lambda-s)
$$

for $\operatorname{Re}(s)>0$. Clearly then $U_{-}^{*}(s)$ can have at most one pole for $\operatorname{Re}(s)>0$ at $s=\lambda$, so that we may write.

$$
U_{-}^{*}(s)=\frac{\alpha}{\lambda-s}+g(s)
$$


where $g(s)$ is same analytic function both for $\operatorname{Re}(s) \leq 0$ and for $\operatorname{Re}(s) \geq 0$, and $\alpha$ is a constant. Furthermore, since $U_{-}^{*}(s)$ and $U_{+}^{*}(s)$ are bounded on their respective damains, so is $g(s)$. Therefore by Liouville's theorem $g$ (s) must be a constant. To obtain it, set $s=0$ in (15) ; since

$$
U_{+}^{*}(0)=A^{*}(0)=S^{*}(0)=K^{*}(0)=1
$$

it follows that $U_{-}^{*}(0)=0$, yielding

$$
g(s)=g(0)=-\alpha / \lambda
$$

Therefore (16) becomes

$$
\mathrm{U}_{+}^{*}(s)\left[s-\lambda+\lambda s^{*}(s)\right]=\frac{\lambda s s^{*}(s)}{s+\mu} U_{+}^{*}(s+\mu)+s \alpha / \lambda
$$

or

$$
U_{+}^{\star}(s)=\frac{\alpha}{\lambda}: m(s)+\ell(s) \cdot U_{+}^{*}(s+\mu), \quad \operatorname{Re}(s) \geq 0
$$

where

$$
\left\{\begin{array}{l}
m(s)=\frac{s}{s+\lambda\left(s^{*}(s)-1\right)} \\
\ell(s)=\frac{\lambda s s^{*}(s)}{(s+\mu)\left[s+\lambda\left(s^{*}(s)-1\right)\right]}
\end{array}\right.
$$

Let us first determine $\alpha$, using the fact that $U_{+}^{*}(0)=1$. After some algebra, necessitated by the indeterminate forms which arise, we have

$$
\alpha=\lambda(1-\lambda E[S])-\frac{\lambda^{2}}{\mu} U_{+}^{*}(\mu)
$$

where $U_{+}^{*}(\mu)$ is still unknown. Notice also that $\frac{\alpha}{\lambda}$ is exactly $U(0)$.

We are now ready to prove the main result of this section. 
Theorem 7. If $\lambda E[S]<1$, then $U_{+}^{*}(s)$ the Laplace-Stieltjes transform of $U(x) \triangleq Y(x) / D(x)$ is given by :

(20) $\quad U_{+}^{*}(s)=U(0) \cdot \xi(s)$

where $\xi(s)=m(s)+\sum_{n=1}^{\infty} m(s+n \mu) \prod_{i=0}^{n-1} \ell(s+i \mu)$

and

$$
U(0)=(1-\lambda E[S]) \cdot \frac{1}{1+\frac{\lambda}{\mu} \cdot \xi(\mu)}
$$

Proof : By applying (17) recursively to itself we obtain for any $\mathrm{N}>0$

$$
U_{+}^{*}(s)=U(0)\left[m(s)+\sum_{n=1}^{i N} m(s+n \mu) \prod_{i=0}^{n-1} \ell(s+i \mu)\right]+\prod_{i=0}^{N} \ell(s+i \mu) \cdot U_{+}^{*}(s+(N+1) \mu)
$$

Notice first that $m(s)$ and $\ell(s)$ are analytic for $\operatorname{Re}(s) \geq 0$.

To show this it suffices to examine their poles for $R e \geq 0$, which are simply the zeros (see (18)) of

$$
s+\lambda\left(s^{*}(s)-1\right)
$$

Now clearly for $\operatorname{Re}(s) \geq 0$

$$
\left|\frac{d}{d s} s^{*}(s)\right| \leq E[S]
$$

so that, if $\lambda E[S]<1$

$$
\left|\lambda \int_{0^{-}}^{s} d s^{*}(s)\right|=\lambda\left|s^{*}(s)-1\right| \leq \lambda E[s]|s|<|s|
$$

Therefore by Rouché's theoren $s+\lambda\left(s^{*}(s)-1\right)$ has exactly the same number of zeros for $\operatorname{Re}(s) \geq 0$ as $s$, namely one at $s=0$, which cancels with the zero of $m(s)$ or of $\ell(s)$. Hence $m(s)$ and $\ell(s)$ have no poles for $\operatorname{Re}(s) \geq 0$ and are thus analytic. Clearly 


$$
\lim _{n \rightarrow \infty}|m(s+n \mu)|=1
$$

while

$$
\lim _{n \rightarrow \infty}\left|U_{+}^{*}(s+n \mu)\right|=U(0)<1
$$

and for same positive constant $K$,

$$
\prod_{i=0}^{n} \ell(s+n \mu) \leq \frac{\lambda^{n}}{\mu^{n}} \frac{K}{n !}
$$

for large enough $n$. Thus as we take $N+\infty$ in (21) the last term vanishes and we remain with $(20)$. The unknown constant $U_{+}^{*}(\mu)$ is determined by solving $(20)$ at $s-\mu$.

A few remarks concerning the form of (17) are worth mentioning.

Remark 6. We see that $\mathrm{m}(\mathrm{s})$ is very similar to the waiting time distribution transform of an $M / G / 1$ queue (it suffices to replace $\alpha / \lambda$ by $(1-\lambda E[S])$ in the mumerator). Therefore $U$ can be viewed as the sum of two random variables, the first of which is similar in form to the waiting time of an $M / G / 1$ system.

Remark 7. As $\mu \rightarrow \infty$ (the delay $D$ is zero), (19) yields

$$
\alpha=\lambda(1-\lambda E[\mathbf{S}])
$$

since $\ell(s)+0$; hence (17) becames

$$
\mathrm{U}_{+}^{*}(s)=\frac{s(1-\lambda E[S])}{s+\lambda\left(S^{*}(s)-1\right)}
$$

which is precisely, as expected, the transform of the waiting time in the $M / G / 1$ system.

More generally since

$$
\mathrm{U}(\mathrm{x})=\mathrm{Y}(\mathrm{x}) / \mathrm{D}(\mathrm{x}), \quad \mathrm{x}>0
$$


it follows that when $D$ is exponentially distributed

$$
Y(x)=U(x)-e^{-\mu x} U(x)
$$

so that

$$
\mathrm{Y}^{*}(\mathrm{~s})=\mathrm{U}_{+}^{*}(\mathrm{~s})-\mathrm{U}_{+}^{*}(\mathrm{~s}+\mu)
$$

From (17) we then have

$$
U_{+}^{*}(s)=U(0) m(s)+\ell(s)\left[U_{+}^{*}(s)-Y^{*}(s)\right]
$$

or

(22) $\quad Y^{*}(s)=U(0) \frac{m(s)}{\ell(s)}+U_{+}^{*}(s)\left[\frac{l(s)-1}{l(s)}\right]$

A special case of interest corresponds to the model analyzed in [5] when we set $S_{n}=0, n \geq 1$. We then have :

Corollary 8 Under the conditions of Theorem 7 , if $s_{n}=0, n \geq 1$, it follows that

$$
U_{+}^{*}(s)=e^{-\lambda / \mu}\left(1+\sum_{n=1}^{\infty} \prod_{i=1}^{n}\left(\frac{\lambda}{s+\mu i}\right)\right)
$$

Remark 8. For $\mu \gg \lambda$ (the average value of $D$ is very small) we have

$$
U_{+}^{*}(s) \cong[1+\lambda /(s+\mu)] /[1+\lambda / \mu]
$$

or

$$
U(x) \cong 1-\frac{\lambda / \mu}{1+\lambda / \mu} e^{-\mu x}, \quad x \geq 0
$$

so that 


$$
Y(x) \cong 1-e^{-\mu x}\left(1+\frac{\lambda / \mu}{1+\lambda / \mu}\right)+e^{-2 \mu x} \frac{\lambda / \mu}{1+\lambda / \mu}
$$

yielding

$$
E[Y] \cong \frac{1}{\mu}\left(1+\frac{\lambda / \mu}{1+\lambda / \mu}\right)-\frac{1}{2 \mu} \cdot \frac{\lambda / \mu}{1+\lambda / \mu}
$$

or

$$
\mathrm{E}[\mathrm{Y}] \cong \frac{1}{\mu}+\frac{1}{2 \mu} \cdot \frac{\lambda / \mu}{1+\lambda / \mu}
$$

Remark 9. The average number of customers in the system just after a departure may be obtained in the case of Poisson arrival using equation (8) which remains satisfied here.

\section{CONCLUSIONS}

Resequencing algorithms have been analyzed in this paper for two types of disordering systems of interest for modelling comunication networks and distributed systems. The case where the disordering delay is an independent increment process, as well as that of independent identically distributed delays, are considered. The approach taken is that of examining the end to end delay encountered by each object in the system. These leads to some new analytical results concerning both the "pure" resequencing problem and concerning the effect of an "output service time" on resequencing delays. 
[1] KLEINROCK L., "Communication Nets : Stochastic Message flow and Delay". Mac Graw-Hill, New York, 1964, reprinted by Dover, New York, 1972.

[2] IE IANN G., "Algorithms for distributed data sharing systems which uses Tickets", Proc. 3rd Berkeley Workshop on Distributed Data Management and Computer Networks. San Francisco, August 1978.

[3] ELIIS C., "Consistency and correctness of a duplicate data base systems", Proc. 6th ACM Symposium on operating system Principles, Nov 77.

[4] GEIENBE E., SEVCIK K.C., "Analysis of update synchronization for multiple copy data bases". Proc. 3rd Berkeley workshop on Distributed Data Management and Computer networks, San Francisco, August 1978.

[5] KLETNROCK L., KAMOUN F. \& MUNTZ R., "Queueing analysis of the reordering issue in a distributed database concurrency control mechanism", $2^{\circ}$ int. Conf. on Distributed Computing Systems, Apr. 81, Versailles, France.

[6] HARRUS G., PLATEAU B., "Queueing analysis of a reordering issue", Rapport de recherche $\mathrm{N}^{\circ}$ 92, LRI 1981, Université de Paris-Sud, Orsay, France.

[7] BACCEIJII F., ZNATI T., "Queueing algorithms with breakdowns in data base modelling", Rapport de recherche $n^{\circ} 50$, Dec. 80, INRIA, France.

[8] FEUVRE J.M., "Queueing analysis of a reordering system", Proceedings of PERFORMANCE 81, North-Holland Pub. Comp., Nov. 1981.

[9] BOROVKOV A.A., "Stochastic processes in queueing theory", Springer Verlag, 1976.

[10] TAKACS L., "Introduction to the theory of queues", Oxford University, press. 1962. 
Imprimé en France

par

l'Institut National de Recherche en Informatique et en Automatique 
